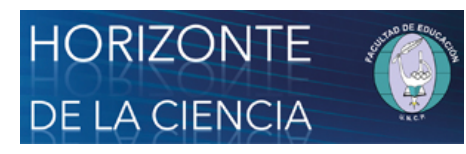

Horizonte de la Ciencia ISSN: 2413-936X

horizontedelaciencia@uncp.edu.pe

Universidad Nacional del Centro del Perú

Perú

\title{
Síndrome de burnout y depresión en trabajadores de la Universidad Nacional del Centro del Perú - 2018
}

\author{
Huachos Pacheco, Aníbal; Veli Chuquillanqui, Rocio \\ Síndrome de burnout y depresión en trabajadores de la Universidad Nacional del Centro del Perú - 2018 \\ Horizonte de la Ciencia, vol. 10, núm. 18, 2020 \\ Universidad Nacional del Centro del Perú, Perú \\ DOI: https://doi.org/10.26490/uncp.horizonteciencia.2020.18.408
}

Los autores otorgan el permiso a compartir y usar su trabajo manteniendo la autoría del mismo. Atribución no comercial (CC BY-NC) 4.0 


\title{
Síndrome de burnout y depresión en trabajadores de la Universidad Nacional del Centro del Perú - 2018
}

\author{
Burnout syndrome and depression in workers of the National University of Central Peru - 2018 \\ Síndrome de Burnout e depressão em trabalhadores da Universidad Nacional del Centro del Peru - 2018 \\ Burnout latachinin lulapaku llakikuynilawan UNCP yachay wasichu Piruchu -2018 \\ BETSIKAKOTANTSI ABISHIMOYETAKERI BURNOUT KANTZIMONETAKERIRI \\ ANTABAIRIANTSIPAYE YOTANTSIPANKOKI NIYANKIBENTSIRORI IRIPERANTSI \\ NANATSINKARITEPAYENI ASHITARO NAMPITSIPE. PITE IRINKA TSA SOTI \\ Kara Okantagitetanaka kara Universidad nacional del centro kara Osarinikë Peru - 2018
}

Aníbal Huachos Pacheco

Universidad Nacional del Centro del Perú, Perú

ahuachosp@gmail.com

(iD http://orcid.org/0000-0002-4545-3527

Rocio Veli Chuquillanqui

Universidad Privada Los Andes, Perú

rocioveli@hotmail.com

iD http://orcid.org/0000-0003-2087-0890
DOI: https://doi.org/10.26490/ uncp.horizonteciencia.2020.18.408

Recepción: 06 Junio 2019 Aprobación: 20 Agosto 2019

Recepción: 06 Junio 2019

Aprobación: 20 Agosto 2019

\section{Resumen:}

La presente investigación comienza con la interrogante ¿Qué relación encontramos entre el Burnout y depresión en trabajadores de la Universidad Nacional del Centro del Perú, durante el año escolar 2018? el motivo que conllevo a este trabajo fue de establecer la correlación entre Burnout y depresión en trabajadores de la Universidad Nacional del Centro del Perú, durante el año escolar 2018. La investigación fue de naturaleza básica. Se utilizó el método descriptivo que permitió constatar la correlación entre el Burnout y la depresión. Se empleó el diseño de investigación descriptivo correccional; El muestreo fue no probabilístico e intencional y para ello se ha trabajado con 150 trabajadores de la Universidad Nacional del Centro del Perú. Los instrumentos que se han utilizado para la investigación son: El Inventario de Maslach y la Escala de Hamilton. En el estudio de los resultados obtenidos se ha utilizado la estadística descriptiva e inferencial. Al final se demostró la existencia de relación entre el agotamiento laboral y la depresión.

Palabras clave: Burnout, Depresión, Trabajador, Universidad, Relación, abishimotakeriri, kantsimotantsi, antabairiantsi, yometantsipankoki, okatsi abishimoyetakeriri, Kenganijitagantsi, antaneri, Universidad, kantaengataro.

\section{Abstract:}

This research begins with the question: What is the relationship between burnout syndrome and depression in workers of the National University of Central Peru, during the academic year 2018? The reason for this work was to establish the correlation between burnout syndrome and depression in workers of the National University of Central Peru, during the academic year 2018. The research was basic in nature. We used descriptive method allowing verifying the correlation between Burnout and depression. The descriptive research design was used corrections; the sampling was non-probability and intentional and to this end we have worked with 150 workers of the National University of Central Peru. The instruments that have been used for research are the inventory of Maslach and the Hamilton scale. In the study of the results obtained has been used the descriptive and inferential statistics. At the end showed the existence of a relationship between job burnout and depression.

\section{Notas DE AUTOR}


KEYWORDS: Burnout, depression, worker, University relationship.

\section{Resumo:}

Esta pesquisa começa com a questão: Que relação encontramos entre Burnout e depressão em trabalhadores da Universidad Nacional do Centro do Peru, durante o ano letivo de 2018? O motivo que levou a este trabalho foi estabelecer a correlação entre Burnout e depressão em trabalhadores da Universidade Nacional do Peru Central, durante o ano letivo de 2018. A pesquisa foi de natureza básica. Utilizou-se o método descritivo que permitiu constatar a correlação entre Burnout e depressão. Foi usado o desenho descritivo da pesquisa correcional; A amostragem não foi probabilística e intencional e, para isso, se trabalhou com 150 trabalhadores da Universidad Nacional do Centro do Peru. Os instrumentos que foram utilizados para pesquisa são: o inventário de Maslach e a escala de Hamilton. No estudo dos resultados obtidos, foram utilizadas estatísticas descritivas e inferenciais. No final, foi demonstrada a existência de uma relação entre exaustão no trabalho e depressão.

Palavras-ChaVe: Burnout, depressão, trabalhador, universidade, relacionamento.

\section{INTRODUCCIÓN}

En estos últimos años el ritmo laboral ha cambiado mucho debido a las exigencias sociales. Ahora se busca profesionales capaces de trabajar bajo presión y sean competentes. En consecuencia, los trabajadores tienen que satisfacer los deseos de sus empleadores y de aquellos a los que brindan servicios, y afrontando las consecuencias que le genera están condiciones a su salud.

Uno de los principales problemas que acontecen en la parte laboral es el Síndrome de Burnout o del quemado, ocasionado por los cambios socioeconómicos y laborales.

Gil-Monte (2002) afirma que el Burnout se da en profesionales que trabajan de manera directa con personas, porque es cansado sobrellevar conductas de otras personas. Una de estas profesiones es la docencia, debido a las instituciones educativas responden al sistema capitalismo caracterizado por la oferta y la demanda, donde se ha olvidado la verdadera naturaleza de la educación y solo que la ve como una empresa que brinda servicios.

Los maestros que tiene que hacer frente a su profesión les generan un agotamiento laboral que afecta su estado psicológico porque no solo se dedican a sus estudiantes, sino también tienen que preocuparse por las actividades administrativas y de investigación que les exige toda entidad educativa superior. Las exigencias laborales hacen que su vida familiar y social sea reducido y no puedan recrease, después de haber realizado agotadas actividades mentales en su centro de trabajo. Todo lo anterior genera en ellos estados de depresión por las constantes condiciones laborales que sobrellevan, además de realizar estudios, hacer su tesis, asistir a un congreso, y buscan tener más resoluciones de felicitaciones para engordar su currículo porque así lo exige la competencia laboral.

Zabalza (2009, págs. 70,71). Afirma que son muchos los maestros que tienen que dejar a su familia para ser un buen profesional, todo esto los acaba, lo agota porque son personas que no disfrutan de la vida plenamente.

El docente universitario no solo tiene que preocuparse por brindar un buen servicio a sus estudiantes, además debe de ser sometido a encuestas estudiantiles cada semestre. Esto les genera preocupación por obtener un buen puntaje y tener mejores oportunidades laborales, pero qué sucede con aquellos que sufren de Burnout y han llegado a la depresión. Son aquellos maestros que inevitablemente van a salir mal en estas encuestas y solo esperan el desenlace de su historial laboral.

El agotamiento laboral lo explican las siguientes teorías psicosociales:

a. Teoría Socio cognitiva del Yo: Según Harrison (1983, pág. 29): Los seres humanos por lo general elegimos carreras profesionales para prestar ayuda a los demás. Están predispuesto a ciertos estados emocionales como la depresión o el estrés producto por los conflictos personales, sobrecarga laboral, etc. Con el tiempo la persona sufre de Burnout.

b. Teoría de Intercambio Social: Esta teoría hace referencia a las relaciones laborales que se tiene con los compañeros de trabajo. Muchos profesionales no piden ayuda a los demás porque amenaza su autoestima y 
piensan que serán criticados. Tienden a comparar su trabajo con la de sus compañeros de trabajo y se sienten pésimos para (Buunk y Schaufeli (1993, pág. 53).

c. Teoría Organizacional: Consideran que los factores que desencadenan el Burnout son: la rutina, monotonía, la falta de organización dentro del centro de trabajo que produce enfrentamientos entre los colegas. Todo lo anterior genera en las personas cansancio laboral lo define (Winnubst, 1993 pg. 151).

Las personas que llegan a tener Burnout tienen problemas físicos, motivacionales y emocionales. El Sahili (2015, pág. 9) afirma que es más peligroso el Burnout que la depresión, porque un docente puede vivir meses y años con desgaste laboral y nadie puede percibirlo, pero en cambio la depresión por caracterizarse por el bajo estado anímico advierte a las personas que lo rodean y ser atendido de manera inmediata.

Expresiones constantes de maestros que padecen de Burnout:

- Me incomoda mis estudiantes porque cada vez están peores y no sé cómo controlarlos.

- No me gusta ir a mi trabajo.

- El personal administrativo no considera que tengo mucho trabajo.

- Tengo miedo de ser despedido.

- Siento que los estudiantes perciben que mi trabajo no es el de antes.

- No puedo organizar mi trabajo de manera adecuada.

Las causas que provocan la existencia de Burnout en los trabajadores:

- Desilusión Gradual del trabajo:

- Menos control en la vida:

- Preferencias a unos en detrimento a otros:

- Agudización de la discrecionalidad laboral: Los trabajadores cuando sienten que sus colegas son más que él y desempeñan el mismo trabajo. Al sentir que sus colegas tienen un mejor horario que el suyo. Sienten que algunos tienen más oportunidades de crecer profesionalmente en el Centro laboral.

La depresión en los Trabajadores es una enfermedad que no es momentánea, que afecta su estado emocional y sobre todo se ha convertido en parte de la vida de todo educador.

Se considera que una persona se encuentra en depresión cuando su tristeza es constante y esto es causado por el estrés. La depresión es la constante tristeza que presenta la persona que afecta el cuerpo y la mente. La persona no come, ni duerme, afecta su autoestima. No pueden decir voy a ponerme mejor porque necesitan de ayuda externa para controlar los sentimientos que tienen.

Factores que pueden ocasionar la depresión

Según San y Arranz (2010, pág. 17):

- Los genes: Se sugiere la existencia de un factor genético que permite heredar la depresión. Se debe tener cuidado si la familia lo padece.

- La personalidad: Las personas obsesivas, rígidas, controladoras y que les gustan las cosas perfectas suelen estar predispuestas a la depresión cuando no consiguen lo que quieren.

- Estilo de pensamiento: Las personas que no celebran sus logros y tiene un pensamiento negativo sobre sí mismo.

- Enfermedades crónicas: Tener alguna discapacidad y ser inseguros desencadenan la depresión.

Estrés y sucesos vitales estresantes: La muerte de un ser querido, pasar por un divorcio, no tener un empleo seguro, la jubilación próxima.

\section{Materiales y MÉTodos}

La investigación se llevó a cabo entre los trabajadores administrativos de la Universidad Nacional del Centro del Perú.

El estudio es descriptivo correlacional se desarrolló por medio del cuestionario que fue aplicado a una muestra de 225 personas entre varones y mujeres durante el año 2018. 
Los trabajadores estuvieron distribuidos de la siguiente manera 98 varones y 127 mujeres, sus edades oscilan entre 18 a 60 años; la condición laboral es de contratados y nombrados, la mayoría de ellos son personal auxiliar, técnico y profesionales; una mínima parte de son funcionarios; en primer lugar, respondieron el inventario de Maslach para Síndrome de Burnout y posteriormente la Escala de depresión de Hamilton.

Los trabajadores fueron seleccionados utilizando la técnica de muestreo de sujetos voluntarios y estuvo comprendida por todos aquellos que se sometieron a la evaluación por su propia voluntad.

Los instrumentos utilizados fueron; para la primera variable el inventario de agotamiento de Maslach; que es uno de los instrumentos más utilizados para medir la presencia de estrés laboral o síndrome de quemado en el trabajo; introducido en 1981, continúa siendo probado en su estructura factorial por diversos autores (Byrne, 1993; Richarsen y Martinussen, 2004; García, Herrero, y León, 2007). Desde 1974 Cristina Maslach reconoce el "Síndrome de Burnout" (Síndrome de estar quemado) en los sujetos afectados por "sobre carga emocional" debido a las características de su trabajo, como una respuesta emocional de los profesionales de ayuda. La primera versión del instrumento fue desarrollada por Maslach y Jackson, en 1981, y la segunda versión, en 1986, esta última es la que se utiliza largamente en las diferentes investigaciones pues se encuentra traducida al castellano.

El Maslach Burnout Inventory (MBI) es un instrumento en el que se plantea al sujeto una serie de enunciados sobre los sentimientos y pensamientos con relación a su interacción con el trabajo.

Es el instrumento que ha generado un mayor volumen de estudios e investigaciones, formado por 22 ítems que se valoran con una escala tipo Likert. El sujeto valora, mediante un rango de 6 adjetivos que van de "nunca" a "diariamente", con qué frecuencia experimenta cada una de las situaciones descritas en los ítems.

Para la variable depresión se utilizó la Escala de Evaluación para la Depresión de Hamilton fue diseñada para ofrecer una medida de la intensidad o gravedad de la depresión. La versión inicial, con 24 items, data de 1960 y fue posteriormente revisada y modificada por el mismo autor, en 1967, reduciéndola a 21 items. De ellos, los cuatro últimos no son útiles a efectos de valorar la intensidad o gravedad de la depresión, por lo que en los ensayos clínicos de evaluación terapéutica se ha generalizado el uso de una versión reducida de 17 items, que corresponden a los 17 primeros de la versión publicada en 1967.

La versión de 21 items fue adaptada al castellano por Conde y cols en 1984, quien introdujo un ítem final para valoración de otros síntomas, por lo que consta de 22 items. Ramos-Brieva y Cordero (1986) adaptaron al castellano y validaron la versión reducida de 17 items.

Para la descripción de los resultados se utilizaron tablas de frecuencias; para establecer la relación entre las variables y sus dimensiones se manejó la correlación tau-b de Kendal, mientras que la contrastación de las hipótesis se ha realizado con la prueba de significancia.

\section{Resultados}

La muestra estuvo conformada por 225 trabajadores, de las facultades de Educación, Enfermería, Ingenierías, Contabilidad, Economía, Arquitectura y Sociología. En los resultados se encontró que 96 servidores que representan al 46,67\% sufren de Burnout, 71 tienen un Burnout moderado y 58 un Burnout bajo. Al revisar los antecedentes se encontró en las investigaciones que los trabajadores sufren de desgaste laboral por el constante estrés en el que se encuentran sometido a día a día en su entorno de trabajo. 
TABLA 1

Niveles del Síndrome de Burnout

\begin{tabular}{|c|c|c|}
\hline Categorí & Escala & Cantidad (fi) $\%(f i)$ \\
\hline $\begin{array}{l}\text { Bajo } \\
\text { Medio } \\
\text { Alto } \\
\text { Total }\end{array}$ & $\begin{array}{rl}\text { Menor de } 34 & 15 \\
34-66 & 51 \\
67-99 & 159 \\
225\end{array}$ & $\begin{array}{l}6,67 \% \\
22.67 \% \\
70,67 \% \\
100,0 \%\end{array}$ \\
\hline
\end{tabular}

Nota: Datos obtenidos después de la aplicación del Inventario de Maslach a los trabajadores de la Universidad Nacional del Centro del Perú, 2018.

En la tabla 1, se puede observar que después de haber aplicado el cuestionario de Maslach para medir el Burnout en una muestra de 225 trabajadores de la Universidad Nacional del Centro del Perú, se encontró que 15 trabajadores de la Universidad Nacional del Centro del Perú (6,67\%) se encuentran en el nivel bajo, 51 trabajadores de la Universidad Nacional del Centro del Perú $(22,67 \%)$ están ubicadas en el nivel medio y 159 trabajadores de la Universidad Nacional del Centro del Perú (70,67\%) se sitúan en el nivel alto, lo que revela que la mayor parte de la muestra encuestada presenta un alto nivel del Síndrome de Burnout.

TABLA 2

Niveles de los resultados de la depresión

\begin{tabular}{llcl}
\hline Categoría & Escala & Cantidad (fi) & $\%$ (fi) \\
No se encuentra deprimido & $0-07$ & 42 & $18.67 \%$ \\
Presenta una depresión menor & $8-13$ & 87 & $36,67 \%$ \\
Presenta una depresión moderada $14-18$ & 45 & $20,00 \%$ \\
Presenta una depresión severa & $19-22$ & 9 & $4.00 \%$ \\
Presenta una depresión severa & $23-52$ & 42 & $18,67 \%$ \\
\multicolumn{1}{l}{ Total } & & 225 & $100 \%$ \\
\hline
\end{tabular}

Nota: Resultados de la escala de Hamilton a los trabajadores de la Universidad Nacional del Centro del Perú, 2018.

En la tabla 2, se puede verificar que después de haber aplicado la escala de Hamilton para saber el nivel de depresión de una muestra de 225 trabajadores de la Universidad Nacional del Centro del Perú, se encontró que 42 trabajadores de la Universidad Nacional del Centro del Perú (18,67\%) no están deprimidos, 87 trabajadores de la Universidad Nacional del Centro del Perú $(38,67 \%)$ presentan una depresión menor, 45 trabajadores de la Universidad Nacional del Centro del Perú (20\%) evidencian una depresión moderada, 9 trabajadores de la Universidad Nacional del Centro del Perú (4\%) muestran una depresión severa y 42 trabajadores de la Universidad Nacional del Centro del Perú $(18,67 \%)$ mostraron tener una depresión muy severa. Según estos datos la tendencia es que la mayor cantidad de encuestados tiende a algún nivel de depresión que va desde el nivel menor hasta muy severa.

La teoría Sociocognitiva del yo es quien afirma que la administración pública por la naturaleza tan delicada y dedicada de los trabajadores con el tiempo sufre de Burnout. En las encuestas, también se evidencio la mayoría de los trabajadores sufren de estrés y no se preocupan por su salud mental y esto aumenta cuando comienzan las matrículas y las labores académicas.

En la aplicación de la entrevista a 225 trabajadores que sufren de Burnout alto, resultó que 116 de ellos que corresponde al 51,67\% sufren de una depresión severa, 53 trabajadores que es el (23.33\%) presentan una depresión moderada y 56 trabajadores $(25.00 \%)$ tiene una depresión menor. La naturaleza de la depresión no es fácil de estudiarlo, pero si revisamos el Modelo Psicodinámico, nos explica que la protección y cariño que recibimos en la infancia es importante, ahora bien, que sabemos nosotros de aquellos maestros que están 
en las aulas universitarias si tuvieron una familia feliz y cariñosa, porque son esos aspectos que influyen en el desarrollo de la depresión más adelante. Además, que sucede si el docente realiza el mayor esfuerzo por cumplir son su trabajo y no recibe estímulos de sus empleadores o más aun de sus estudiantes, lo afirma el Modelo Conductual, donde el docente se siente mal porque piensa que su trabajo no es bueno y esto lo lleva al aislamiento.

De todo ello se puede afirmar que los trabajadores que tienen problemas son los contratados, del sexo masculino, divorciados y están en la edad de 41-50 años.

Cuando se relacionó las variables de Burnout y depresión se evidenció que existe una relación fuerte entre ambas variables, es ahí donde se reafirma que los trabajadores que tienen mayor desgaste laboral su nivel de depresión es mayor.

\section{Discusión}

Se observa que gran parte de la muestra encuestadas los docentes sufren de Burnout, lo que se justifica porque, la naturaleza de su trabajo y las actividades que realizan es agotadora, extenuante y llena de responsabilidad y dedicación, como se explica en el marco teórico, Maslach y Jackson (1981) se preocuparon por investigar los factores laborales que causan la existencia del Burnout. En este caso las jornadas de trabajo exigentes, inseguras, con alta presión, y llenos de incertidumbre conducen a las personas a sufrir altos niveles de estrés crónico y luego sentir la sensación de estar agotado, consumido o quemado. Los efectos no son los mismos ya que algunas personas dadas las características de su personalidad son más propensas a sufrir depresión, respecto a otras que tienen mejores estrategias de afrontamiento.

Los resultados arrojan que la presencia de cansancio emocional es elevada en los trabajadores de la Universidad Nacional del Centro del Perú.

Respecto a la variable depresión, se detectó que el 77.34\% de la muestra tiene algún grado de depresión que va desde aquellos que no padecen hasta el nivel moderado; por otra parte un $22.64 \%$ de la población de la muestra encuestada tiene un nivel de depresión entre grave y severamente grave lo cual es preocupante, ya que según Rehm, Gordon-Leventon e Ivens (1987), el estrés hace que a persona afecta se sienta triste o "vació", o realiza las actividades que le gusta, sufre de insomnio, no tiene ganas de comer o viceversa, se aleja de sus amigos, presenta baja autoestima y tiene pensamientos suicidas.

Al correlacionar agotamiento emocional, despersonalización y autorrealización de la variable de Burnout con la variable depresión se evidencia que todas las dimensiones muestran correlaciones positivas y bajas con la depresión, pero todas son significativas, lo que evidencia que hay una asociación de la variable Síndrome de Burnout con la variable depresión y de todas las dimensiones de Síndrome de Burnout con la variable depresión, lo cual es congruente con las investigaciones y resultados.

En nuestro medio son pocas las investigaciones que se han realizado acerca del Burnout que padecen los docentes, producido por las exigencias físicas, psicológicas y sobre todo sociales. Se debe mencionar, además, las nuevas leyes implementadas por los Gobiernos de turno para evaluar al docente.

La prevalencia de SBO en trabajadores de la Universidad Nacional del Centro del Perú, durante el año lectivo 2018, fue de un 34,2\%, que se comparó con el trabajo de investigación de Mariño, Otero, Málaga (2011), que obtuvieron un resultado de $13,4 \%$.

Los puntajes que puedan resultar después de la aplicación del MBI tiene una fácil interpretación, con ayuda del Manual sugerido por Maslach y Jackson et al. (1981)

Castaño, García, Leguizamón (2006) en su investigación concluyeron que las personas que padecen de Burnout son jóvenes, mujeres, solteros y que tiene mayores turnos de trabajo y tienen una personalidad idealista, con expectativas elevadas y buen ingreso económico.

Con esta investigación se demostró que existe una relación parcial entre el Síndrome de Burnout y la Depresión en el trabajador universitario. El personal de sexo masculino que sufren un alto nivel de Burnout 
y depresión severa; presentan problemas con la familia y los amigos; mientras que el servidor administrativo que no tiene una familia constituida se refugia en su trabajo para sentirse útiles, también sufren de depresión fuerte en comparación a los casados.

\section{REFERENCIAS BIBLIOGRÁFICAS}

Buunk, B. y Schaufeli, W. (1993). Burnout: una perspectiva de la teoría de comparación social. En W. B. Schaufeli, C. Maslach, y T. Marek (Eds.), Serie en psicología aplicada: cuestiones y preguntas sociales. Burnout profesional: desarrollos recientes en teoría e investigación. Filadelfia, Pensilvania, EE. UU: Taylor y Francis.

Castaño, García y Leguizamón (2006) Asociación entre el síndrome de estrés asistencial en residentes de medicina interna, El reporte de sus prácticas médicas de cuidados su óptimos y reporte de los pacientes, Univ. Psychol. Bogotá (Colombia) 5 (3): 549-561.

El Sahili, L. (2015). Burnout. Consecuencias y Soluciones. Distrito Federal, México: Editorial el Manual Moderno.

Gil - Monte, P. (2002). Validez Factorial de la adaptación al español del Maslach Burnout Inventory - General Survey. Scielo Salud pública. Recuperado el 23 de agosto de 2018, de http://www.insp.mx/salud/index.html

Harrison, W. D. (1983). A social competence model of burnout. En: Farber, B. A. (Ed.), Stress and burnout in the. New York: Pergamon Press.

Mariño, A., Otero, M., Málaga, G. y Tomateo, J. (2011). Coexistencia del síndrome de Burnout y síntomas depresivos en médicos residentes. Estudio descriptivo transversal en un Hospital Nacional de Lima. Revista Médica Herediana

Maslach, C. \& Jackson, S. (1981). The measurement of experienced burnout. Journal of Occupational Behavior, $19,99,113$.

Ramos-Brieva y Cordero (1986) Un procedimiento breve y rápido para medir la intensidad de los síntomas depresivos en atención primaria. Scopus, Actas Esp Psiquiatr 2011;39(1):45-8.

Rehm, Gordon-Leventon e Ivens (1987), Rehm. Depression. En C. L. Frame y J. L. Matson (Eds.), Handbook of assessment in childhood 341-371). New York, NY: Plenum Publishing Corp. Stevenson, psychopathology (pp. D. T.

San y Arranz (2010). Comprender la Depresión. Barcelona: Editorial Amat S.L.

Winnubst, J. (1993). Organizational structure, social. London: Taylor.

Zabalza, M. (2009). Ser un profesor universitario hoy. La cuestión universitaria, 70.

Los autores otorgan el permiso a compartir y usar su trabajo manteniendo la autoría del mismo.

CC BY-NC 